\title{
Acquired immunodeficiency syndrome and the health care worker
}

\author{
SAMUEL KRACHMAN, DO
}

GLBERT E. D'ALONZO, JR., DO

The risk of acquiring $\mathrm{HIV}$ infection for the health care worker is low but increased compared to the general population ( $5 \%$ versus $3 \%$, respectively). The osteopathic physician from every size community and urban hospital must be informed and prepared to answer questions regarding the most effective methods to prevent transmission of HIV to the health care worker. Additionally, the osteopathic physician must also be able to instruct the health care worker in the proper management of accidental parenteral and mucosal exposure to the blood and body fluids of a patient.

Human immunodeficiency virus (HIV), the etiologic agent for acquired immunodeficiency syndrome (AIDS), has been isolated from a number of body fluids, excretions, and secretions, including urine, cerebrospinal fluid, semen, blood, vaginal secretions, saliva, tears, amniotic fluid, and breast milk. Epidemiologic studies, however, have implicated transmission to occur only through sexual contact, exposure to blood or blood products, and perinatally from an infected mother to her infant.

With the increasing prevalence of patients with AIDS and AIDS-related conditions in community and urban hospitals, the health care worker is likely to be at increased risk of exposure to the blood products and secretions of these infected patients. As of July $1987,5.8 \%$ of the 32,395 adult cases of AIDS were in patients employed in a health care or clinical laboratory setting. ${ }^{1}$ Of these, $95 \%$ belonged to a high-risk group, but the remaining
$5 \%$ had an undetermined means of HIV acquisition. Health care workers with AIDS were significantly more likely than other workers to have an undetermined risk ( $5 \%$ versus $3 \%$, respectively) for infectivity.

The occupational risk of acquiring HIV infection for hospital personnel has been shown to be low; it is estimated to be less than $1 \%$ in those who have had parenteral contact with blood from infected patients. $^{2}$

The purpose of this article is to provide information for the osteopathic physician on the risks and prevention of HIV infectivity for the health care worker. This information hopefully will be useful in establishing precautionary guidelines in osteopathic hospitals and other health care facilities to give the health care worker optimal protection.

\section{Risks of contagion \\ Risks in a health care setting}

A number of prospective studies of exposed health care workers have been carried out to assess the risk of nosocomial transmission of HIV (Table 1). As of June 1987, the Centers for Disease Control (CDC), ${ }^{1}$ in an ongoing study, prospectively evaluated 883 health care workers who had a documented percutaneous exposure to blood $(708,80 \%)$ or mucous membrane or open wound contamination by blood or body fluids $(175,20 \%)$ of HIVinfected patients. Of the total study population, 396 $(45 \%)$ had a convalescent phase serum sample evaluated 90 days after exposure. One worker for whom heterosexual transmission could not be ruled out was seropositive. Additionally, 425 workers had acute- and convalescent-phase serum samples evaluated; three with percutaneous exposure seroconverted (none had additonal risk factors). ${ }^{1}$ 
Weiss and associates ${ }^{3}$ studied 361 health care workers from several metropolitan area hospitals. Six of the 23 workers who were categorized into a high-risk group were HIV positive. Three of the 361 health care workers had possible parenteral exposure and were HIV-positive, but had no known associated risk factors: One had sustained sticks from needles used to draw blood from an HIVpositive patient on two separate occasions. Another had punctured her finger with a forceps used on an HIV-infected patient. Heterosexual transmission could not be ruled out, because her sexual partner also was found to be seropositive. The third recalled handling blood from a multiply transfused leukemic patient and cut his hand.

Henderson and coworkers ${ }^{4}$ prospectively studied 531 health care workers. Of these, 150 had reported percutaneous or mucous membrane exposure to blood or body fluids from patients with AIDS. None of these were seropositive on follow up from 6 to 46 months after the recorded exposure. Of the other 381 participants in the study (all of whom had no exposure to blood or body fluids), three were seropositive, but each belonged to at least one of the high-risk groups.

Lifson and coresearchers ${ }^{5}$ looked at occupational information of $16,748 \mathrm{HIV}$-seropositive patients reported to the CDC as of May 1, 1986; $922(5.5 \%)$ were identified as health care workers. Of these, 88 did not belong to groups at increased risk for AIDS. Forty-four of the 61 for whom follow up information was available eventually were reclassified into a high-risk group, leaving only 17 of those followed up and 27 of those without further data (44 total) with no known risk factors. Thus, $95 \%$ of all health care workers reported by this study belonged to high-risk groups. When individuals from countries where heterosexual transmission may play a major role were excluded, the proportion of cases with no identifiable risk among health care workers $(3.7 \%)$ did not differ significantly from the proportion of cases with no identified risk among nonhealth workers $(2.8 \%)$.

A study in Zaire also has shown a lack of association between HIV seroprevalence in hospital workers and occupational exposure. Of the 2,384 hospital workers studied by Mann and associates, ${ }^{6}$ 152 workers $(6.4 \%)$ were seropositive for HIV. Seropositivity was significantly higher among women and unmarried persons. Three personal medical factors were associated significantly with HIV seropositivity: blood transfusion, hospitalization during the previous ten years, and receiving parenteral injections during the previous three years. Seropositivity was not shown to be associated with reported occupational exposures to HIV-infected pa- tients or their blood products. It is important to note that information about sexual practices was not evaluated by this study.

Thus, the risk of HIV transmission in the health care setting is low. With the additional implementation of precautionary guidelines, the risk should be reduced further.

\section{Risks in an AIDS household setting}

Other data to support the low incidence of the nonsexual transmission of HIV comes from studies of household members of infected patients. Friedland and coauthors ${ }^{7}$ analyzed the household contacts of 39 patients, 37 with AIDS and two with AIDSrelated complex (ARC). The household contacts had no sexual interactions with the patients and no other risk factors for AIDS. They did, however, share items and facilities, such as eating utensils, drinking glasses, showers, and toilets, with the patients. In this study, despite prolonged and close association with AIDS patients, 100 of 101 household contacts remained free of infection. The one person who became infected, a 5-year-old child whose mother had AIDS, had had symptoms and signs of infection since infancy. Therefore, the only household infection in this study appeared to have been acquired by perinatal transmission.

In a study by Fischl and associates, ${ }^{8}$ the incidence of HIV infection among heterosexual partners, children, and household contacts of patients with AIDS was examined. Of the 29 adult household contacts who were enrolled in the study, none had HIV seroconversion. Of the 15 children with AIDS, ARC, or generalized persistent lymphadenopathy, all were younger than 4 years and all were children of HIV-infected mothers. This evidence of an absence of horizontal transmission of $\mathrm{HIV}$ infection should reduce the fears and concerns of an ever-growing population of close contacts and household members of patients with AIDS, as well as of the population at large.

\section{Prevention of transmission to health care workers}

\section{Universal precautions}

It is difficult and unreliable to depend on a history and physical examination to identify patients with $\mathrm{HIV}$ infection. Thus, the $\mathrm{CDC}^{1}$ has recommended the use of "universal precautions," so that blood and body fluid precautions will be used consistently on all patients. This especially applies to the emergency care setting, where the risk of exposure to blood and blood products is increased. Six major universal precautions are as follows:

(1) The routine use of barrier precautions to pre- 
develop AIDS before the mother even becomes symptomatic. The risk of AIDS in the newborn of an $\mathrm{HIV}$-infected pregnant woman is high-at least $50 \% .{ }^{9}$ An infected newborn in the delivery suite exposes the medical and nursing staff to blood and secretions that are potentially infective. Universal precautions, as well as those observed in all operating rooms, should be followed. Also, the pediatrician who will care for a potential HIV-infected neonate should be informed, so as to employ precautions.

The $\mathrm{CDC}^{10}$ has reported a case of apparent transmission of HIV from an infected child to a mother caring for that child. The mother was exposed to stool, urine, blood, and other body fluids and did not wear any gloves.

Autopsy or mortician's service. In addition to the universal precautions, all persons performing postmortem procedures should wear gloves, masks, protective eyewear, gowns, and waterproof aprons. Instruments should be decontaminated along with all surfaces used following each procedure.

Ophthalmologic examination. In 1985 , scientists at the National Institutes of Health ${ }^{11}$ were able to isolate HIV from the tears of a patient with AIDS. The patient had no eye complaints at the time. Professionals who perform eye examinations and other procedures involving contact with tears should practice good handwashing technique after and before each procedure and between each patient. The use of gloves is advisable, especially when cuts, scratches, or dermatologic lesions are present on the hands.

Instruments that come into direct contact with external surfaces of the eye should be wiped clean and disinfected. Contact lenses used in trial fittings should be disinfected between each fitting, usually with a hydrogen peroxide solution for both hard and soft lenses.

Dialysis. Techniques used for disinfecting hemodialysis machines consist of methods aimed at controlling bacterial contaminations. ${ }^{1}$ Generally, 500 $750 \mathrm{ppm}$ of sodium hypochlorite (household bleach) for 30 to 40 minutes or $1.5 \%$ to $2.0 \%$ formaldehyde overnight are used. There also are several commercially available germicides for disinfecting the fluid pathways of the hemodialysis machine. None of these procedures needs to be changed after dialyzing an HIV-infected patient.

Peterman and associates ${ }^{12}$ studied the incidence of transmission of HIV among hemodialysis patients at eight Chicago-area chronic dialysis centers. Twenty-five $(4.8 \%)$ of 520 hemodialysis pa- tients screened for HIV by the enzyme immunoassay (EIA) were positive. Of these, four were strongly reactive and had an associated positive result with the Western Blot method; the other 21 were weakly reactive by EIA and had a negative Western Blot test.

The weakly reactive EIA results were associated with antibodies against the H9 lymphoid cell line, which is the same cell line used to propagate the HIV used in the EIA test. Some of the H9-cellassociated antigens can contaminate the purified HIV. Dialysis patients with a low reactivity EIA had received significantly more blood transfusions as compared with seronegative controls. The researchers concluded that there was no evidence of transmission of HIV infection in the dialysis centers.

Of the four positive patients, one was infected by a blood transfusion, and another had an identified risk factor other than blood product transfusion. The two others had no documented cause, but both probably received transmission by one of their many blood transfusions.

The hemodialyzers of HIV patients can be discarded after use. Alternatively, a dialyzer can be reused on the same patient only; the dialyzer is issued to a specific patient, removed, cleaned, and disinfected and reused on that patient several times.

Laboratories and blood banks. Beside the universal precautions, the $\mathrm{CDC}^{1}$ has outlined specific precautions for health care workers in the laboratory. All specimens of blood or body fluids that are collected should be placed in well-constructed containers with secure lids to prevent leaking during transportation. Biologic safety cabinets or hoods need only be used when a procedure carries a high potential for droplet formation, such as blending or vigorous mixing. Mechanical, not mouth pipetting, should be performed for all liquid specimens in the laboratory. Specific attention should be paid to the decontamination of work surfaces and scientific equipment. Workers should wash their hands after handling any specimen.

General recommendations. The standard sterilization and disinfection procedures appear to be adequate when used for instruments and devices contaminated by products from HIV-infected patients. Studies ${ }^{13-15}$ have shown that commonly used germicides rapidly inactivate $\mathrm{HIV}$, even at concentrations that are much lower than those used in present hospital practice. Embalming fluids have been shown to be equally effective in inactivation of HIV. In addition to the commercially available 
germicidal agents, common household bleach (sodium hypochlorite) in concentrations ranging from $1: 100$ to $1: 10$ dilution is effective in inactivating HIV. Manufacturer's recommendations should be followed, because sodium hypochlorite can cause corrosion with repeated use on certain medical devices.

Instruments that enter sterile tissue or the vascular system should be sterilized. Devices that come in contact with mucosal surfaces should be sterilized or treated with a high-level disinfectant. Medical devices should be cleaned thoroughly before being sterilized or disinfected. Chemical germicides that are registered as "sterilants" with the Environmental Protection Agency may be used either to sterilize or disinfect, depending upon the contact time.

Housekeeping practices in the hospital need not be altered, because transmission of HIV from walls, floors, and other surfaces has not been shown to occur. The routine cleaning methods using disinfectant detergent formulations seem to be adequate and effective. Decontaminating spills of blood and body fluids in the patient-care setting involves removal of the visible material, followed by decontamination. In contrast, with spills of cultured or concentrated infectious agents in the laboratory, the involved area should be flooded with a liquid germicide first. The area should then be cleaned and again decontaminated with germicide.

The risk of HIV transmission by handling soiled laundry is likely to be negligible. All soiled linen should be bagged at the location where it is used. Linen that is soiled with blood and body fluids should be placed in leak-resistant bags.

\section{Management of health care workers after HIV exposure}

\section{Serologic testing}

Adults infected with HIV usually will develop antibodies to the virus within 6-12 weeks after exposure. The sensitivity and specificity of the currently used EIA is acceptable when performed on serum specimens from HIV-positive persons who have been infected for longer than 12 weeks. ${ }^{1}$

A specimen that initially is reactive by EIA should have a repeat test performed to avoid laboratory error. The Western Blot test then is used to increase the specificity of repeatedly reactive EIA results. This testing sequence is highly predictive of HIV infection. ${ }^{1}$ If the Western Blot result is equivocal, the test is repeated; if it is still indeterminant, the test should be repeated again in three to six months. ${ }^{1}$

\section{Infected health care worker}

HIV-infected health care workers could be at increased risk of contracting infectious diseases and their complications secondary to a potentially impaired immune system. Therefore, any HIV-positive health care workers should receive counseling regarding this potential risk, especially if they are involved in caring for patients with transmissible infections. These workers should continue to follow the standard infectious control recommendations to minimize the risk of acquiring another infection.

Finally, the question arises whether these HIVpositive health care workers can be allowed to continue to perform their duties without increased risk to patients. Although no case of transmission of HIV from infected health care workers performing invasive procedures on their patients has been reported, this decision should be made on an individual basis. The medical and department directors and the health care worker's personal physician should be involved in the decision.

\section{Management of exposure}

Even though the risk of transmission of HIV by percutaneous needlestick or mucous membrane exposure is low, it is still present and probably is of primary concern to the health care worker involved. If a health care worker has an accidental parenteral or mucous membrane exposure to blood or body fluids, or has serious cutaneous exposure involving large volumes of blood for a prolonged time period, the patient involved should be told of the accident and asked for consent to be tested seriologically for HIV.

If the patient has a history of AIDS, is HIV positive, or refuses consent for the test, the worker should be tested for evidence of HIV infection. If the results are negative, the worker should be retested at 6 weeks and again on a periodic basis (such as at 12 weeks and 6 months) to rule out transmission.

However, if the patient from whom the blood or body fluid originated is seronegative, no further follow up is needed unless the patient has a known risk factor. If this is the case, a specimen from the health care worker at 12 weeks can be obtained to rule out transmission.

\section{Conclusions}

Osteopathic physicians need to be prepared to answer questions regarding the most effective techniques and methods that can be implemented to prevent transmission of HIV to the health care worker. In addition, questions regarding the man- 
agement of accidental parenteral or mucosal exposure to the blood or body fluids of a patient must be addressed. These answers should reflect the latest CDC recommendations as well as the data on risk of infectivity, as reviewed in this article.

The increasing prevalence of HIV in this country forces the osteopathic physician in every size community or urban practice to be familiar with the disease process and its variable presentations. While it appears that the osteopathic physician has the medicolegal right to accept or refuse an individual patient who asks for treatment, ${ }^{16}$ most physicians would agree that there is an ethical responsibility to treat and help people who are in need of care-including the AIDS patient.

Along these same lines, the osteopathic physician feels the responsibility to protect fellow health care workers from the potential risk of infectivity that can occur in caring for these patients. We hope that this article will help the osteopathic physician to meet this responsibilty and that it will assist in answering some of the many questions regarding this growing national health care concern.

1. Recommendation for prevention of HIV transmission in health care settings. MMWR 1987;36(Suppl):3S-18S.

2. McCray E. Occupational risk of the acquired immunodeficiency syndrome among health care workers. N Engl J Med 1986;314:1127-32.

3. Weiss SH, Saxinger WC, Rechtman D, et al. HTLV-III infection among health care workers: Association with needle stick injuries. JAMA 1985;254:2089-93.

4. Henderson DK, Saah AJ, Zak BJ, et al: Risk of nosocomial infection with human T-cell lymphotropic virus type III/lymphadenopathy-associated virus in a large cohort of intensively exposed health care work- ers. Ann Intern Med 1986;104:644-647.

5. Lifson AR, Castro KG, McCray E, et al: National surveillance of AIDS in health care workers. JAMA 1986;256:3231-3234.

6. Mann JM, Francis H, Quinn TC, et al: HIV seroprevalence among hospital workers in Kinshasa, Zaire. Lack of association with occupational exposure. JAMA 1986;256:3099-3102.

7. Friedland GH, Saltzman BR, Rogers MF, et al: Lack of transmission of HTLV-III/LAV infection to household contacts of patients with AIDS or AIDS-related complex with oral candidiasis. $N$ Engl $\mathrm{J} \mathrm{Med}$ 1986;314:344-349.

8. Fischl MA, Dickinson GM, Scott GB, et al: Evaluation of heterosexual partners, children, and household contacts of adults with AIDS. JAMA 1987;257:640-644.

9. Ledger WA: AIDS and the obstetrician/gynecologist: Commentary, in AIDS: Information on AIDS for the Practicing Physician. Chicago, American Medical Association, 1987, vol 2, pp 5-6.

10. Apparent transmission of human T-lymphotropic virus type III/LAV from a child to a mother providing health care. MMWR 1986;35:76-79.

11. Recommendations for preventing possible transmissions of HTLV III/LAV virus from tears. MMWR 1985;(Suppl)4:24.

12. Peterman TA, Lang GR, Mikos NJ, et al: HTLV-III/LAV infection in hemodialysis patients. JAMA 1986;255:2324-2326.

13. Spire B, Montagnier L, Barré-Sinoussi F, Chermann JC: Inactivation of lymphadenopathy associated virus by chemical disinfectants. Lancet $1984 ; 2: 899-901$.

14. Martin LS, McDougal JS, Loskoski SL: Disinfection and inactivation of the human T lymphotropic virus type III/lymphadenopathyassociated virus: $J$ Infect Dis 1985;152:400-403.

15. McDougal JS, Martin LS, Cort SP, et al: Thermal inactivation of acquired immunodeficiency syndrome virus III/ymphadenopathy associated virus with special reference to antihemophilic factor. J Clin Invest $1985 ; 76: 875-877$.

16. Zuger A, Miles SH: Physicians, AIDS, and occupational risk: Historic traditions and ethical obligations. JAMA 1987;258:1924-1928.

From the Pulmonary Division, Department of Internal Medicine, the University of Texas Health Science Center at Houston, Houston, Tex.

For reprints, address Dr Krachman, the University of Texas Health Science Center at Houston, 6431 Fannin, Suite 1.274, Houston, TX 77030 . 